\title{
Nasometric values for European Portuguese: preliminary results
}

Isabel Falé ${ }^{1,3}$ and Isabel Hub Faria ${ }^{1,2}$

${ }^{1}$ Laboratório de Psicolinguística, Universidade de Lisboa, Portugal

${ }^{2}$ DLGR, Faculdade de Letras, Universidade de Lisboa, Portugal

${ }^{3}$ Universidade Aberta, Portugal

https://doi.org/10.36505/ExLing-2008/02/0022/000081

\begin{abstract}
Nasal sounds frequencies in European Portuguese represent $21 \%$ of Português Fundamental corpus sounds (Nascimento et al. 1987), revealing how nasality plays an important role in this language and how a speech problem affecting nasality can interfere severely in one's speech intelligibility. In order to obtain the first standard nasometric values for European Portuguese we developed two tests (syllable repetition and text reading) and collected data from 25 adults. Preliminary results showed that: oral stimuli achieved an average nasalance score of $10 \%$; syllables with nasal consonant and nasal vowel achieved $77 \%$ and the reading passages with nasal saturation presented an average score of $44 \%$. Considering these results, we acknowledge the existence of three different levels of normal nasality.
\end{abstract}

Key words: nasometry, nasality, speech production, speech assessment

\section{Introduction}

Resonance is one of the most prominent characteristics of human speech. Problems affecting nasal resonance are widely reported in speech pathologies, causing severe perturbations in subjects speech intelligibility.

Nasal resonance values can be obtained through different instrumental methods, however, the nasometer from Kay Elemetrics 6200-3 proved to be an indirect and objective assessment instrument which results have shown to have a high correlation with perceived nasality (Hardin et al. 1992, Hirschberg et al. 2005). This nasometer provides a nasalance score which corresponds to a ratio of nasal to nasal-plus oral acoustic energy, multiplied by 100 .

Normative nasalance scores have been already determined for several languages such as English (Seaver et al. 1991), French, Spanish (Nichols 1999), Puerto Rican Spanish (Anderson 1996), Finnish (Haapanen 1991), Dutch, Flemish (van Lierde et al. 2000) and Hungarian (Hirschberg et al. 2005). Some of the studies pointed to the existence of differences among different dialects, reinforcing the need to have nasalance norms established for each sample in every language.

Our research main goal was to collect normative nasalance scores for European Portuguese language, in order to make possible an easy detection

ExLing 2008: Proceedings of 2nd Tutorial and Research Workshop on Experimental Linguistics, 25-27 August 2008, Athens, Greece 
of nasal resonance problems in this language, either hiponasality or hipernasality.

\section{Experiment}

In European Portuguese there are three nasal consonants and five nasal vowels. Usually, nasal consonants spread their nasal feature to the adjacent vowels nasalizing them. Considering these language specificities, we developed two tests: a repetition syllable test and a text reading test.

\section{Experimental material}

Starting from one sub-test of the Mackay-Kummer Test (SNAP) - repetition syllable test - we built a new one, the TN-PE, which raised the number of pre-existent syllables in order to assess language specific consonant-vowel combinations, namely those with nasal vowels. Forty syllables distributed by four combinations of resonance type syllables were considered: A. oral consonant - oral vowel (13 syllables); B. oral consonant - nasal vowel (8 syllables); C. nasal consonant - oral vowel (12); D. nasal consonant - nasal vowel (7).

We also created eight reading passages considering two basic different criteria: absence of nasal sounds and saturation of nasal sounds (26 to 33\% of nasal sounds in each). Neither of them was balanced in respect to the presence of nasals in speech continua in European Portuguese. They were only designed to detect hipernasality and hiponasality productions, respectively.

\section{Subjects}

25 adult (12 females) subjects EP native speakers, aged 19 to 27, with no history of otorhinolaryngological disorders, abnormal nasality or without common colds and nasal congestion participated in this preliminary study.

\section{Nasometry and the nasometer}

For this study we used the Nasometer 6200-3 da Kay Elemetrics which is a computer-based system, where the oral and nasal acoustic energy components are captured by microphones mounted on each side of a separator plate, that is placed in the subject's upper lip for data collecting.

The nasometer was calibrated before data collecting and the position of the headset was adjusted according to manufacturer's manual specifications in order to get reliable data. 


\section{Experimental procedure}

The syllable-repetition test required participants to repeat a consonant-vowel syllable six to ten times in two seconds.

The text reading test required the participants to read a small text with no hesitations or pauses. Each time a participant made a reading mistake or made a longer pause he had to start over and read the text again. For experimental purposes, we only considered the well read texts.

\section{Results and discussion}

Normal nasalance score for stimuli with no nasal segments is in average $10 \%$, both in syllable repetition and in text reading (see Table 1 and Table 2). Nasalance values different from zero in oral stimuli were also reported in studies from other languages (see Hirschberg et al. 2005 for a literature review). Results of syllable types B and C are very near from each other, especially if we consider the high standard deviation values that both present. This may mean that nasal consonants and nasal vowels are quite similar in what acoustic energy is concerned. The highest nasalance score average was achieved in the syllable type $\mathrm{D}$. This value is higher than the ones for other languages with values ranging between $40 \%$ and $60 \%$. However, not every other language has nasal vowels in its phonological system so when the values for nasals are shown they are considering only nasal consonants with oral vowels like our syllable-type C.

Nasalance scores for text reading saturated with nasals sounds are considerably lower than the ones registered in syllable repetition test and closer to scores from other languages.

Table 1. Results of nasalance scores by Resonance Syllable type.

\begin{tabular}{|l|l|l|}
\hline Syllable - types & Average & Standard deviation \\
\hline A - OO & $10 \%$ & 2,83 \\
\hline B - ON & $69 \%$ & 12,22 \\
\hline C - NO & $65 \%$ & 9,74 \\
\hline D - NN & $77 \%$ & 9,45 \\
\hline
\end{tabular}

Table 2. Results of nasalance scores by Text Reading.

\begin{tabular}{|l|l|l|}
\hline Text Reading & Average & Standard deviation \\
\hline No nasals & $10 \%$ & 3,15 \\
\hline Nasals saturation & $44 \%$ & 8,07 \\
\hline
\end{tabular}

Taking into account the results of this experiment we acknowledge the existence of three levels of normal nasality in European Portuguese: N0 corresponds to a level of nasality of $10 \%$ for oral stimuli (syllable and text); 
$\mathrm{N} 1$ is the intermediate level of nasality - $40 \%$ - and corresponding to text reading saturated with nasals; and N2, the highest level, corresponds to $70 \%$ of nasalance and characterizes the syllable-type D (nasal consonant and nasal vowel). The difference between each level is of 30\% starting from the lowest average nasalance score for oral stimuli.

\section{Conclusions}

This research provided the first global nasalance scores for European Portuguese in a syllable-repetition task and in a text reading task. Results revealed to be quite similar to values from other languages, especially in what oral stimuli are concerned.

Based on these results, we proposed the existence of three levels of normal nasality for European Portuguese: N0, N1 and N2.

Two forthcoming studies, one with 66 EP native children (aged 6 to 10) and the other with 30 females, will provide further evidence for this proposal.

\section{References}

Anderson, R. T. 1996. Nasometric values for normal Spanish-speaking females: a preliminary report. Cleft Palate-Craniofacial Journal, 33, 333-336.

Haapanen, M.L. 1991. Nasalance scores in normal Finnish speech. Folia Phoniatrica e Logopaedia, 43, 197-203.

Hardin, M. et al. 1992. Correspondence between nasalance scores and listener judgments of hypernasality and hyponasality. Cleft Palate-Craniofacial Journal, $29,346-351$

Hirschberg et al. 2005. Adaptation of nasometry to Hungarian language and experiences with its clinical application. International Journal of Pediatric Otorhinolaryngology, 70, 5, 785-798.

Mueller, K. et al. 2007. Diagnostic value of nasometry - representative study of patients with cleft palate and normal subjects. Folia Phoniatrica et Logopaedia, 59, 219-226.

Nascimento, F. et al. 1987. Português Fundamental. Vol. II, tomo 1, Lisboa: INIC, CLUL.

Nichols, A. 1999. Nasalance statistics for two Mexican populations. Cleft PalateCraniofacial Journal, 36, 57-63.

Seaver, E. et al. 1991. A study of nasometric values for normal nasal resonance. Journal of Speech and Hearing Research 34, 715-721.

Van Lierde, K. M. et al. 2000. Nasometric values for normal nasal resonance in the speech of young Flemish adults. Cleft Palate-Craniofacial Journal, 38, 112-118. 